\title{
The Poisson Burr X Inverse Rayleigh Distribution And Its Applications
}

\author{
Rania H. M. Abdelkhalek ${ }^{*}$ \\ Department of Statistics, Mathematics and Insurance, Benha University, Egypt.
}

\begin{abstract}
A new flexible extension of the inverse Rayleigh model is proposed and studied. Some of its fundamental statistical properties are derived. We assessed the performance of the maximum likelihood method via a simulation study. The importance of the new model is shown via three applications to real data sets. The new model is much better than other important competitive models.
\end{abstract}

Keywords: Inverse Rayleigh; Poisson; Simulation; Modeling.

*rania.abdelkhalek@fcom.bu.edu.eg 


\section{Introduction and physical motivation}

The well-known inverse Rayleigh (IR) model is considered as a distribution for a life time random variable (r.v.). The IR distribution has many applications in the area of reliability studies. Voda (1972) proved that the distribution of lifetimes of several types of experimental (Exp) units can be approximated by the IR distribution and studied some properties of the maximum likelihood estimation (MLE) of the its parameter. Mukerjee and Saran (1984) studied the failure rate of an IR distribution. Aslam and Jun (2009) introduced a group acceptance sampling plans for truncated life tests based on the IR. Soliman et al. (2010) studied the Bayesian and nonBayesian estimation of the parameter of the IR model Dey (2012) mentioned that the IR model has also been used as a failure time distribution although the variance and higher order moments does not exist for it.

The probability density function (PDF) and cumulative distribution function (CDF) of the IR distribution are given by (for $x \geq 0$ )

$$
g_{I R}^{(\alpha)}(x)=2 \alpha^{2} x^{-3} \exp \left[-\alpha^{2} x^{-2}\right] \text { and } G_{I R}^{(\alpha)}(x)=\exp \left[-\alpha^{2} x^{-2}\right]
$$

respectively, where $\alpha>0$ is a scale parameter. It is worth mentioning that the IR model is a special case for the Inverse Weibull model.

In the statistical literature, there are many useful extensions of the IR model, for example: beta IR by Barreto-Souza et al. (2011), Marshall-Olkin IR by Krishna et al. (2013), Transmuted IR by Mahmoud and Mandouh (2013), Weibull IR by Afify et al. (2016), odd Lindley IR by Korkmaz et al. (2017), Burr X IR by Yousof et al. (2017a), transmuted ToppLeone IR by Yousof et al. (2017b), Topp-Leone Generated IR by Yousof et al. (2018b) and odd log-logistic IR Yousof et al. (2018b), among others.

In this paper we propose and study a new extension of the IR distribution using the zero truncated Poisson (ZTP) distribution. Suppose that a system has $N$ subsystems functioning independently at a given time where $N$ has ZTP distribution with parameter $\lambda$. It is the conditional probability distribution of a Poisson-distributed r.v., given that the value of the r.v. is not zero. The probability mass function (PMF) of $N$ is given by

$$
P M F_{Z T P}^{\lambda}(N=n)=\left[\exp (-\lambda) \lambda^{n}\right] /\left.\left\{n !\left[\tau_{\lambda}\right]\right\}\right|_{n=1,2, \ldots}
$$

Note that for ZTP r.v., the expected value $\mathbf{E}(N \mid \lambda)$ and variance $\operatorname{Var}(N \mid \lambda)$ are, respectively, given by

where $\tau_{(\lambda)}=-\exp (-\lambda)+1$ and

$$
\mathbf{E}(N \mid \lambda)=\lambda / \tau_{(\lambda)}
$$

$$
\operatorname{Var}(N \mid \lambda)=-\frac{\lambda^{2}}{\left[\tau_{(\lambda)}\right]^{2}}+\frac{\lambda+\lambda^{2}}{\tau_{(\lambda)}}
$$

Suppose that the failure time of each subsystem has the $\operatorname{Burr} X \operatorname{IR}(B X-\operatorname{IR}(\theta, \alpha)$ for short) defined by the CDF and PDF given by 
and

$$
H_{B X-I R}^{\theta, \alpha}(x)=\left[1-\exp \left(-\left\{\frac{\exp \left[-\alpha^{2} x^{-2}\right]}{1-\exp \left[-\alpha^{2} x^{-2}\right]}\right\}^{2}\right)\right]^{\theta}
$$

$$
\begin{aligned}
h_{B X-I R}^{\theta, \alpha}(x)= & 4 \theta \alpha^{2} x^{-(2+1)}\left\{1-\exp \left[-\alpha^{2} x^{-2}\right]\right\}^{-3} \times\left[1-\exp \left(-\left\{\frac{\exp \left[-\alpha^{2} x^{-2}\right]}{1-\exp \left[-\alpha^{2} x^{-2}\right]}\right\}^{2}\right)\right]^{\theta-1} \\
& \times \exp \left[-2\left(\alpha x^{-1}\right)^{2}\right] \exp \left(-\left\{\frac{\exp \left[-\alpha^{2} x^{-2}\right]}{1-\exp \left[-\alpha^{2} x^{-2}\right]}\right\}^{2}\right)
\end{aligned}
$$

respectively, where $\alpha>0$ is a scale parameter and $\theta>0$ is the shape parameter. Let $Y_{i}$ denote the failure time of the ith subsystem and let

$$
X=\min \left\{Y_{1}, Y_{2}, \cdots, Y_{N}\right\} \text {. }
$$

Then the conditional CDF of $X$ given $N$ is

$$
F(x \mid N)=1-\operatorname{Pr}(X>x \mid N)=1-\left[1-H_{B X-I R}^{\theta, \alpha}(x)\right]^{N}
$$

Therefore, the unconditional CDF of the Poisson BX-IR (PBX-IR) density function, as described in Ristić and Nadarajah (2014), can be expressed as

then we have

$$
F_{P B X-I R}^{\lambda, \theta, \alpha}(x)=\frac{1-\exp \left(-\lambda H_{B X-I R}^{\theta, \alpha}(x)\right)}{\tau_{\lambda}}
$$

$$
F_{P B X-I R}^{\lambda, \theta, \alpha}(x)=\frac{1-\exp \left(-\lambda\left[1-\exp \left(-\left\{\frac{\exp \left[-\alpha^{2} x^{-2}\right]}{1-\exp \left(-\alpha^{2} x^{-2}\right)}\right\}^{2}\right)\right]^{\theta}\right)}{\tau_{\lambda}}
$$

the corresponding PDF is

$$
\begin{aligned}
f_{P B r X I W}^{(\lambda, \theta, \alpha)}(x) & =\frac{4 \theta \lambda 2 \alpha^{2}}{\tau_{(\lambda)}} x^{-(2+1)}\left\{1-\exp \left[-\alpha^{2} x^{-2}\right]\right\}^{-3} \\
& \times \exp \left(-2\left(\alpha x^{-1}\right)^{2}-\left\{\frac{\exp \left[-\alpha^{2} x^{-2}\right]}{1-\exp \left[-\alpha^{2} x^{-2}\right]}\right\}^{2}\right) \\
& \times\left[1-\exp \left(-\left\{\frac{\exp \left[-\alpha^{2} x^{-2}\right]}{1-\exp \left[-\alpha^{2} x^{-2}\right]}\right\}^{2}\right)\right]^{\theta-1} \\
& \times \exp \left\{-\lambda\left[1-\exp \left(-\left\{\frac{\exp \left[-\alpha^{2} x^{-2}\right]}{1-\exp \left[-\alpha^{2} x^{-2}\right]}\right\}^{2}\right)\right]^{\theta}\right\}
\end{aligned}
$$

The transformation of Ristić and Nadarajah (2014) used by many authors such as Ramos et al. (2015), Aryal and Yousof (2017) and Korkmaz et al. (2018), among others. 
The hazard rate function (HRF) can be easily calculated via $f_{P B r X I W}^{(\lambda, \theta, \alpha)}(x) /[1-$ $\left.F_{P B X-I R}^{(\lambda, \theta, \alpha)}(x)\right]$. The PBX-IR density can be right-skewed, unimodal and symmetric (see Figure 1) whereas the PBX-IR HRF can be unimodal then bathtub, increasing and bathtub (see Figure 2).
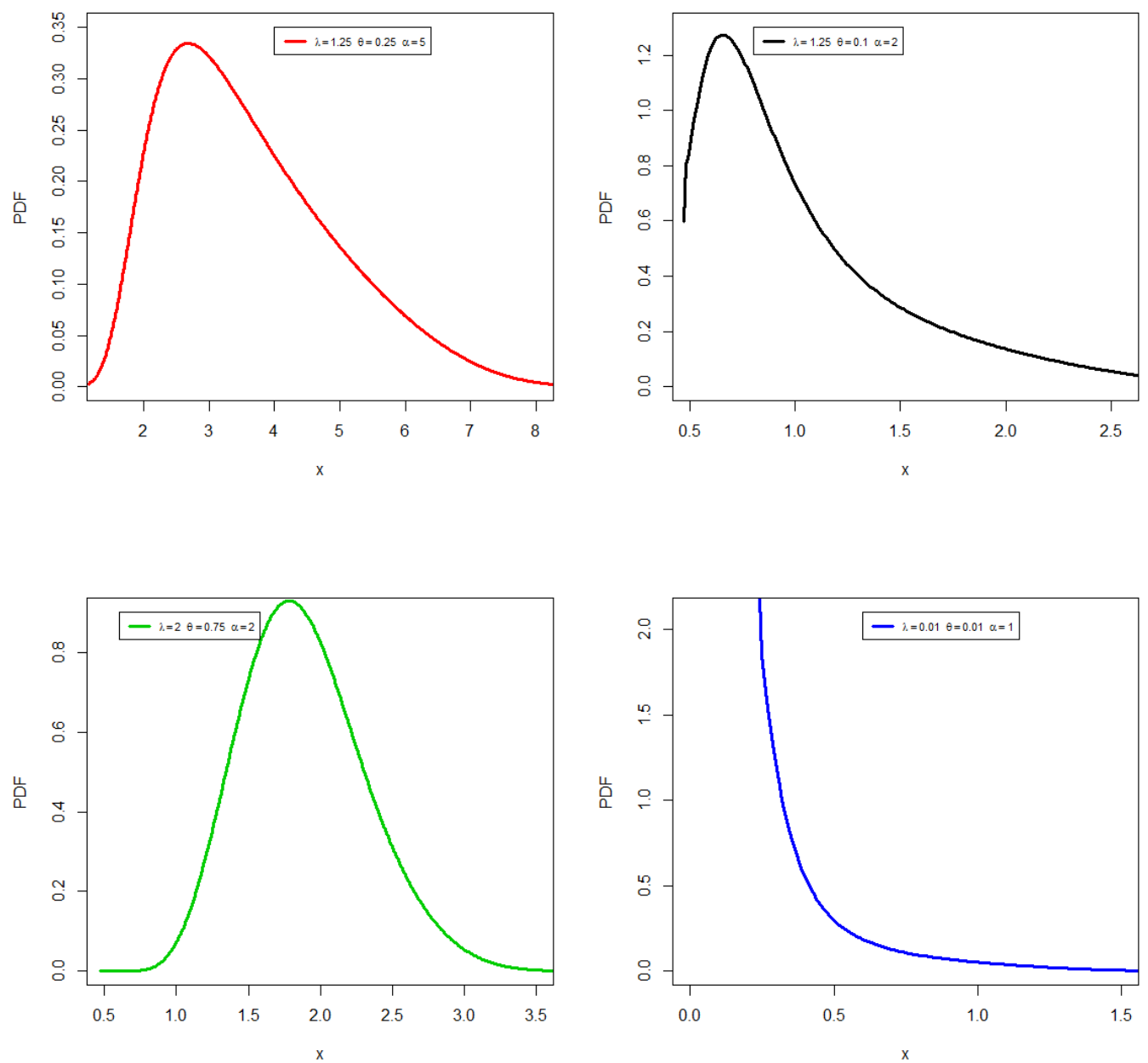

Figure 1: Plots of the PBX-IR PDF for selected parameter values. 

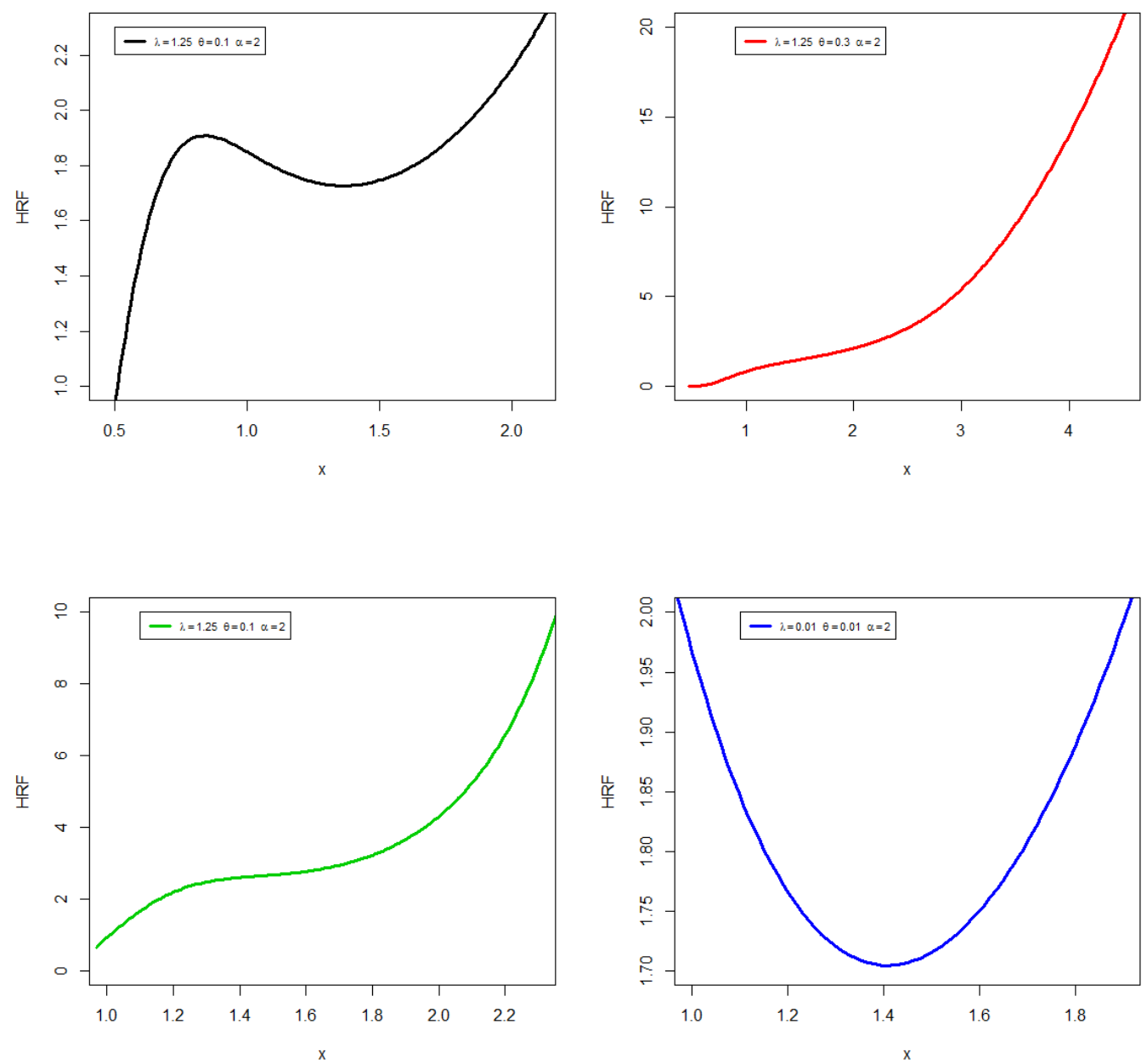

Figure 2: Plots of the PBX-IR HRF for selected parameter values.

This article is organized as follows: In Section 2, we derive some mathematical properties of the new model. Maximum likelihood method for the model parameters is addressed in Section 3. Sections 4 presents the simulation studies. In Section 5, The potentiality of the proposed model is illustrated by means of three real data sets. Section 6 provides some concluding remarks.

\section{Mathematical properties}

\subsection{Useful expansions}

Upon the power series

$$
\exp (\zeta)=\sum_{q=0}^{\infty}\left(\frac{\zeta^{q}}{q !}\right)
$$


the PDF in (6) can be written as

$$
\begin{aligned}
f_{P B r X I W}^{(\lambda, \theta, \alpha)}(x) & =\sum_{q=0}^{\infty} \frac{2 \theta \lambda^{1+h} 2 \alpha^{2}(-1)^{h}}{h !\left[\tau_{(\lambda)}\right]} \\
& \times \exp \left(x^{-(2+1)} \frac{\exp \left[-\alpha^{2} x^{-2}\right]}{\left\{1-\exp \left[-\alpha^{2} x^{-2}\right]\right\}^{3}}\right) \\
& \times \exp \left[-\left(\frac{\exp \left[-\alpha^{2} x^{-2}\right]}{1-\exp \left[-\alpha^{2} x^{-2}\right]}\right)^{2}\right] \\
& \times\left[1-\exp \left(-\left\{\frac{\exp \left[-\alpha^{2} x^{-2}\right]}{1-\exp \left[-\alpha^{2} x^{-2}\right]}\right\}^{2}\right)\right]^{\theta(h+1)-1}
\end{aligned}
$$

If $|\zeta|<1$ and $\tau>0$ is a real non-integer, the following power series holds

Applying (8) to (7) we have

$$
(1-\zeta)^{\tau}=\sum_{i=0}^{\infty} \frac{(-1)^{i} \Gamma(1+\tau)}{\Gamma(1+\tau-i)} \zeta^{i}
$$

$$
\begin{aligned}
f_{P B r-I R}^{(\lambda, \theta, \alpha)}(x) & =\frac{2 \theta 2 \alpha^{2} x^{-(2+1)} \exp \left[-2 \alpha^{2} x^{-2}\right]}{\left[\tau_{(\lambda)}\right]} \\
& \times \sum_{h, i=0}^{\infty} \frac{\lambda^{1+h}(-1)^{h+i} \Gamma(\theta(h+1))}{\mathrm{i} ! \Gamma(\theta(h+1)-i)} \\
& \times \frac{\exp \left[-(i+1)\left(\frac{\exp \left[-\alpha^{2} x^{-2}\right]}{1-\exp \left[-\alpha^{2} x^{-2}\right]}\right)^{2}\right]}{\left[1-\exp \left[-\alpha^{2} x^{-2}\right]\right]^{3}}
\end{aligned}
$$

Via applying the power series to the term

equation (9) becomes

$$
\exp \left[-(i+1)\left(\frac{\exp \left[-\alpha^{2} x^{-2}\right]}{1-\exp \left[-\alpha^{2} x^{-2}\right]}\right)^{2}\right]
$$

$$
\begin{aligned}
f_{P B X-I R}^{(\lambda, \theta, \alpha)}(x)= & \sum_{h, i, \omega=0}^{\infty} \frac{4 \alpha^{2} \theta \lambda^{1+h}(-1)^{h+i+\omega}(i+1)^{\omega} x^{-3}}{i ! \omega !\left[\tau_{(\lambda)}\right]} \\
& \times \frac{\Gamma(\theta(\mathrm{h}+1))}{\Gamma(\theta(\mathrm{h}+1)-\mathrm{i})} \frac{\exp \left[-\alpha^{2} x^{-2}\right]\left\{\exp \left[-\alpha^{2} x^{-2}\right]\right\}^{2 \omega+1}}{\left\{1-\exp \left[-\alpha^{2} x^{-2}\right]\right\}^{2 \omega+3}}
\end{aligned}
$$

Then consider the series expansion 


$$
(1-\zeta)^{-c} I_{|\zeta|<1, c>0}=\sum_{i=0}^{\infty} \frac{\Gamma(c+\kappa)}{\kappa ! \Gamma(c)} \zeta^{\kappa}
$$

Applying the expansion in (11) to (10) for the term $\left\{1-\exp \left[-\alpha^{2} x^{-2}\right]\right\}^{2 \omega+3}$, equation (10) becomes

$$
\begin{aligned}
& f_{P B X-I R}^{(\lambda, \theta, \alpha)}(x)={ }_{h, i, \omega, \kappa=0}^{\infty} \frac{2 \theta \lambda^{1+h}(-1)^{h+i+\omega}(i+1)^{\omega}}{i ! \omega ! \kappa !\left[\tau_{(\lambda)}\right][2(1+\omega)+\kappa]} \\
& \times \frac{\Gamma(\theta(h+1)) \Gamma(3+2 \omega+\kappa)}{\Gamma(\theta(h+1)-i) \Gamma(2 \omega+3)}[2(1+\omega)+\kappa] \\
& \times 2 \alpha^{2} x^{-(2+1)} \exp \left\{-[2(1+\omega)+\kappa]\left(\alpha x^{-1}\right)^{2}\right\} .
\end{aligned}
$$

This can be written as

where

$$
f_{P B X-I R}^{(\lambda, \theta, \alpha)}(x)=\sum_{\omega, \kappa=0}^{\infty} v_{\omega, \kappa} h_{[2(1+\omega)+\kappa]}(x ; \alpha)
$$

$$
v_{\omega, \kappa}=\frac{2 \theta \lambda^{1+h}(-1)^{\omega} \Gamma(3+2 \omega+\kappa)}{\omega ! \kappa !\left[\tau_{(\lambda)}\right] \Gamma(2 \omega+3)[2(1+\omega)+\kappa]} \sum_{h, i=0}^{\infty} \frac{(-1)^{h+i} \Gamma(\theta(h+1))(i+1)^{\omega}}{i ! \Gamma(\theta(h+1)-i)}
$$

and $h_{[2(1+\omega)+\kappa]}(x ; \alpha)$ is the IR density with scale parameter $\alpha[2(1+\omega)+\kappa]^{\frac{1}{2}}$. Similarly, the CDF of the PBX-IR model can also be expressed as

$$
F_{P B X-I R}^{(\lambda, \theta, \alpha)}(x)=\sum_{\omega, \kappa=0}^{\infty} v_{\omega, \kappa} H_{[2(1+\omega)+\kappa]}(x ; \alpha),
$$

where $H_{[2(1+\omega)+\kappa]}(x ; \alpha)$ is the the IR density with scale parameter $\alpha[2(1+\omega)+\kappa]^{\frac{1}{2}}$.

\subsection{Quantile and random number generation}

The quantile function (QF) of $X$, where $X \sim \operatorname{PBX}-\operatorname{IR}(\lambda, \theta, \alpha)$, is obtained by inverting (5) as

$$
Q(u)=\alpha\left\{-\ln \left[\left(1+\left\{-\ln \left[1-\left(\frac{-\ln \left\{1-u\left[\tau_{(\lambda)}\right]\right\}}{\lambda}\right)^{\frac{1}{\theta}}\right]\right\}^{\frac{1}{2}}\right)\right]\right\}^{-\frac{1}{2}},
$$

so, simulating the PBX-IR r.v. is straightforward (see Section 4). If $U$ is a uniform variate on the unit interval $(0,1)$, then the r.v. $X=Q(U)$ follows $(6)$.

\subsection{Moments}

The $r^{\text {th }}$ ordinary moment of $X$, say $\mu_{r}^{\prime}$, follows from (12) as 
$\left.\mu_{r}^{\prime}\right|_{(r<2)}=\mathbf{E}\left(X^{r}\right)=\sum_{\omega, \kappa=0}^{\infty} v_{\omega, \kappa} \alpha^{r}[2(1+\omega)+\kappa]^{\frac{r}{2}} \Gamma\left(1-\frac{r}{2}\right)$,

where

$$
\left.\Gamma(1+\boldsymbol{\tau})\right|_{\left(\tau \in \mathbb{R}^{+}\right)}=_{w=0}^{\boldsymbol{\tau}-1}(\boldsymbol{\tau}-w)=\int_{0}^{\infty} x^{\boldsymbol{\tau}} \exp (-t) d x=\boldsymbol{\tau} !,
$$

upon setting $r=1$ in (15) gives the mean of $X$ as

$$
\mathbf{E}(X)=\sum_{\omega, \kappa=0}^{\infty} v_{\omega, \kappa} \alpha[2(1+\omega)+\kappa]^{\frac{1}{2}} \Gamma\left(1-\frac{1}{2}\right) .
$$

\subsection{Incomplete moments}

The $r^{\text {th }}$ incomplete moment of $X$ is defined by

$$
m_{r}(t)=\int_{-\infty}^{t} x^{r} f(x) d x
$$

Based on (12) we can write

$$
\left.m_{r}(t)\right|_{(r<2)}=\sum_{\omega, \kappa=0}^{\infty} v_{\omega, \kappa} \alpha^{r}[2(1+\omega)+\kappa]^{\frac{r}{2}} \gamma\left(1-\frac{r}{2},\left(\frac{\alpha}{t}\right)^{2}\right),
$$

where

$$
\begin{aligned}
\left.\gamma(\boldsymbol{\tau}, q)\right|_{(\boldsymbol{\tau} \neq 0,-1,-2, \ldots)}=\int_{0}^{q} t^{\boldsymbol{\tau}-1} \exp (-t) d t \\
=\frac{q^{\boldsymbol{\tau}}}{\boldsymbol{\tau}}\left\{{ }_{1} \mathbf{F}_{1}[\boldsymbol{\tau} ; \boldsymbol{\tau} a+1 ;-q]\right\} \\
=\sum_{\kappa=0}^{\infty} \frac{(-1)^{\kappa}}{\kappa !(\boldsymbol{\tau}+\kappa)} q^{\boldsymbol{\tau}+\kappa}
\end{aligned}
$$

is the incomplete gamma function.Via setting $r=1$ in (16) gives the $1{ }^{\text {st }}$ incomplete moment of $X$ as

$$
m(t)=\sum_{\omega, \kappa=0}^{\infty} v_{\omega, \kappa} \alpha[2(1+\omega)+\kappa]^{\frac{1}{2}} \Gamma\left(1-\frac{1}{2},\left(\frac{\alpha}{t}\right)^{2}\right) .
$$

The moment generating function of $X$, say $M(t)=\mathbf{E}(\exp (t X))$, is obtained from (12) as $\left.M(t)\right|_{(r<2)}=\sum_{\omega, \kappa, r=0}^{\infty} v_{\omega, \kappa}\left(t^{r} / r !\right) \alpha^{r}[2(1+\omega)+\kappa]^{\frac{r}{2}} \Gamma\left(1-\frac{r}{2}\right)$.

\subsection{Moments of residual life (MRL) function}

The $n^{t h} \operatorname{MRL}\left[c_{n}(t)\right]$, denoted by

$$
c_{n}(t)=\mathbf{E}\left[\left.(X-t)^{n}\right|_{[X>t, n=1,2, \ldots]}\right],
$$

which uniquely determine the $F(x)$. The $n^{\text {th }}$ MRL of $X$ is given by

$$
c_{n}(t)=\frac{1}{1-F(t)} \int_{t}^{\infty}(x-t)^{n} d F(x),
$$


so, we can write

$$
\begin{gathered}
c_{n}(t)=\frac{1}{1-F(t)} \sum_{r=0}^{\infty} \sum_{\omega, \kappa=0}^{\infty} \frac{(-1)^{n-i} n ! t^{n-i}}{i ! \Gamma(n-i+1)} v_{\omega, \kappa} \\
\times \alpha^{n}[2(1+\omega)+\kappa]^{\frac{n}{2}} \Gamma\left(1-\frac{n}{2},\left(\frac{\alpha}{t}\right)^{2}\right)
\end{gathered}
$$

where

$$
\begin{gathered}
\left.\Gamma(\zeta, z)\right|_{(z>0)}=\int_{0}^{z} t^{\zeta-1} \exp (-t) d t \\
\sim\left[1+\frac{\zeta-1}{z}+\frac{(\zeta-1)(\zeta-2)}{z^{2}}+\ldots\right] \frac{z^{\zeta-1}}{\exp (z)}, \\
\Gamma(\boldsymbol{\tau}, q)+\gamma(\tau, q)=\Gamma(\boldsymbol{\tau})
\end{gathered}
$$

and ${ }_{1} \mathbf{F}_{1}[\because,, \cdot]$ is a confluent hypergeometric function.by setting $n=1$, we get the mean residual life function $\left[c_{n=1}(x)\right]$ or the life expectation at age $x$

$$
c_{1}(x)=\mathbf{E}\left[\left.(X-x)\right|_{(X>t, n=1)}\right]
$$

which represents the expected additional life length for the system which is alive at age $x$.

\subsection{Moments reversed residual life (MRRL) functions}

The $n^{\text {th }}$ MRRL $\left[C_{n}(t)\right]$ denoted by

$$
C_{n}(t)=\mathbf{E}\left[(t-X)^{n}\right] \underset{[X \leq t, t>0 \text { and } n=1,2, \ldots]}{,}
$$

which also uniquely determines the $F(x)$. Then, the $C_{n}(t)$ can be formulated as

so that, the $n^{\text {th }}$ MRRL of $X$

$$
C_{n}(t)=\frac{1}{F(t)} \int_{0}^{t}(t-x)^{n} d F(x),
$$

$$
\begin{aligned}
& C_{n}(t)= \frac{1}{F(t)} \sum_{i=0}^{n} \sum_{\omega, \kappa=0}^{\infty} \frac{(-1)^{i} n !}{i !(n-i) !} v_{\omega, \kappa} \\
& \times \alpha^{n}[2(1+\omega)+\kappa]^{\frac{n}{2}} \gamma\left(1-\frac{n}{2},\left(\frac{\alpha}{t}\right)^{2}\right),
\end{aligned}
$$

by setting $n=1$ in $C_{n}(t)$, we get the mean inactivity time (MIT) or mean waiting time (MWT) which also called the mean reversed residual life function

$$
C_{1}(t)=\left.\mathbf{E}\left[(t-X)^{n}\right]\right|_{[X \leq t, t>0 \text { and } n=1]},
$$

which represents the waiting time elapsed since the failure of the system on condition that this failure had occurred in $(0, x)$.

\section{Estimation}

Consider a random sample from your PBX-IR, then the log likelihood function can be expressed as

$$
\log L=n \log 2+n \log \theta+n \log \lambda+n \log 2+2 n \log \alpha-n \log \left[\tau_{(\lambda)}\right]
$$




$$
\begin{aligned}
& -(2+1) \sum_{i=1}^{n} \log x_{i}-3 \log \left(1-q_{i}\right) \\
& +2 \sum_{i=1}^{n} \log q_{i}-\lambda \sum_{i=1}^{n}\left[1-\exp \left(-m_{i}\right)\right]^{\theta}-\sum_{i=1}^{n} m_{i}+(\theta-1) \sum_{i=1}^{n} \log \left[1-\exp \left(-m_{i}\right)\right]
\end{aligned}
$$

where

$$
q_{i}=\exp \left[-\alpha^{2} x_{i}^{-2}\right] \text { and } m_{i}=\left(\frac{q_{i}}{1-q_{i}}\right)^{2} .
$$

The maximum likelihood (ML) method and its procedures are available in the literature with details.

\section{Simulation studies}

Upon (14), we simulate the PBX-IR model by taking $n=20,50,150,500$ and 1000 . For each sample size, we evaluate the ML estimations (MLEs) of the parameters using the optim function of the R software (see the R code in the Appendix). Then, we repeat this process 1000 times and compute the averages of the estimates (AEs) and mean squared errors (MSEs). Table 1 gives all simulation results. The values in Table 1 indicate that the MSEs of $\hat{\lambda}, \hat{\theta}$ and $\hat{\alpha}$ decay toward zero when $n$ increases for all settings of $\lambda, \theta$ and $\alpha$, as expected under first-under asymptotic theory. The AEs of the parameters tend to be closer to the true parameter values (I: $\lambda=0.5, \theta=1.5$ and $\alpha=2.5$ and II: $\lambda=1.5, \theta=0.5$ and $\alpha=1.5$ ) when $n$ increases. This fact supports that the asymptotic normal distribution provides an adequate approximation to the finite sample distribution of the MLEs. Table 1 gives the AEs and MSEs based on 1000 simulations of the PBX-IR distribution for some

\begin{tabular}{|c|c|c|c|c|c|c|c|}
\hline $\mathrm{n}$ & & $\Theta$ & $\overline{\mathrm{AE}}$ & MSE & $\Theta$ & $\mathrm{AE}$ & MSE \\
\hline \multirow[t]{3}{*}{20} & I & $\lambda$ & 0.55310 & 0.66153 II & $\lambda$ & 0.56726 & 0.44319 \\
\hline & & $\theta$ & 1.74321 & 0.31496 & $\theta$ & 0.68213 & 0.39565 \\
\hline & & $\alpha$ & 2.67919 & 0.52371 & $\alpha$ & 1.68324 & 0.51892 \\
\hline \multirow[t]{3}{*}{50} & & $\lambda$ & 0.54450 & 0.49542 & $\lambda$ & 1.55312 & 0.33789 \\
\hline & & $\theta$ & 1.60778 & 0.30532 & $\theta$ & 0.60434 & 0.19345 \\
\hline & & $\alpha$ & 2.55982 & 0.35973 & $\alpha$ & 1.60910 & 0.39672 \\
\hline
\end{tabular}
values of $a$ and $b$ when by taking $n=20,50,150,500$ and 1000 .

Table 1: The AEs, biases and MSEs based on 1000 simulations. 


\begin{tabular}{|c|c|c|c|c|c|c|}
\hline \multirow[t]{3}{*}{150} & $\lambda$ & 0.52349 & 0.39439 & $\lambda$ & 1.51789 & 0.11567 \\
\hline & $\theta$ & 1.53891 & 0.22560 & $\theta$ & 0.55670 & 0.09982 \\
\hline & $\alpha$ & 2.51567 & 0.12589 & $\alpha$ & 1.53457 & 0.27569 \\
\hline \multirow[t]{3}{*}{500} & $\lambda$ & 0.50582 & 0.09117 & $\lambda$ & 1.50328 & 0.01569 \\
\hline & $\theta$ & 1.50874 & 0.00869 & $\theta$ & 0.51320 & 0.04763 \\
\hline & $\alpha$ & 2.50177 & 0.01678 & $\alpha$ & 1.50569 & 0.03810 \\
\hline \multirow[t]{3}{*}{1000} & $\lambda$ & 0.50071 & 0.00315 & $\lambda$ & 1.50029 & 0.00026 \\
\hline & $\theta$ & 1.50089 & 0.00015 & $\theta$ & 0.50025 & 0.00612 \\
\hline & $\alpha$ & 2.50001 & 0.00005 & $\alpha$ & 1.50004 & 0.00024 \\
\hline
\end{tabular}

\section{Real data modeling}

This section presents two applications of the new distribution using real data sets. We shall compare the fit of the new distribution with the Weibull Inverse Weibull (W-IW), exponentiated IW (E-IW), Kumaraswamy IW(Kum-IW), beta IW (B-IW) transmuted IW (TIW), gamma extended IW (GE-IW), Marshall-Olkin IW (MO-IW), MOKum-IW, generalized MO-IW(GMO-IW), KumMO-IW and IW distributions. The PDFs of the competitive model are available in statistical literature. The unknown parameters of the above PDFs are all positive real numbers except for the T-IW distribution for which $|a| \leq 1$. The $1^{\text {st }}$ data set consists of 100 observations of breaking stress of carbon fibres (in Gba) given by Nichols and Padgett (2006). The $2^{\text {nd }}$ data set consists of 63 observations of the strengths of $1.5 \mathrm{~cm}$ glass fibres [see Smith and Naylor (1987)], originally obtained by workers at the UK National Physical Laboratory. Unfortunately, the units of measurement are not given in the paper. The $3^{\text {rd }}$ data set (gauge lengths of $20 \mathrm{~mm}$ ) [see Kundu and Raqab (2009)] consists of 74 observations. In order to compare the distributions, we consider the following criteria: the $-2 \hat{\ell}$ (Maximized Log-Likelihood), AIC (Akaike Information Criterion), CAIC (Consistent Akaike Information Criterion), BIC (Bayesian information criterion) and HQIC (Hannan-Quinn information Criterion). These statistics are given by

$$
\begin{gathered}
A I C=-2 \hat{\ell}+2 \kappa, \\
B I C=-2 \hat{\ell}+\kappa \log (n), \\
H Q I C=-2 \hat{\ell}+2 \kappa \log [\log (n)]
\end{gathered}
$$

and 


$$
C A I C=-2 \hat{\ell}+2 \kappa n /(n-\kappa-1),
$$

Table 2: The statistics AIC;BIC;HQIC and CAIC values for breaking stress data.

\begin{tabular}{lllll}
\hline Model & \multicolumn{5}{c}{ Measures } \\
& AIC & BIC & HQIC & CAIC \\
\cline { 2 - 5 } PBX-IR & 113.2 & 121 & 116.3 & 113.4 \\
PBX-IW & 122.6 & 133.1 & 126.8 & 123.1 \\
W-IW & 294.5 & 304.9 & 298.7 & 294.9 \\
E-IW & 295.7 & 303.5 & 298.9 & 296.0 \\
Kum- & 297.1 & 307.5 & 301.3 & 297.5 \\
IW & & & 315.4 & 311.6 \\
B-IW & 311.1 & 321.6 & 31.6 \\
GE-IW & 312.0 & 332.4 & 316.2 & 312.4 \\
IW & 348.3 & 353.5 & 350.4 & 348.4 \\
T-IW & 350.5 & 358.3 & 353.6 & 350.7 \\
MO-IW & 351.3 & 359.1 & 354.5 & 351.6 \\
\hline
\end{tabular}

Table 3: MLEs and their standard errors (in parentheses) for breaking stress of carbon fibre data.

\begin{tabular}{lllll}
\hline Model & \multicolumn{5}{c}{ Measures } \\
& & & \\
PBX-IR $(\lambda, \theta, \alpha)$ & 5.657 & 0.159 & 3.927 & \\
& $(0.9282)$ & $(0.0132)$ & $(0.0034)$ & \\
$\operatorname{PBX}-\mathrm{IW}(\lambda, \theta, \alpha, \beta)$ & 4.900 & 3.452 & 1.0310 & 0.742 \\
& $(1.247)$ & $(1.024)$ & $(0.193)$ & $(0.117)$ \\
$\mathrm{W}-\mathrm{IW}(\alpha, \beta, a, b)$ & 2.2231 & 0.355 & 6.9721 & 4.9179 \\
& $(11.409)$ & $(0.411)$ & $(113.811)$ & $(3.756)$ \\
$\operatorname{Kum}-\mathrm{IW}(\alpha, \beta, a, b)$ & 2.0556 & 0.4654 & 6.2815 & 224.18 \\
& $(0.071)$ & $(0.00701)$ & $(0.063)$ & $(0.164)$ \\
$\operatorname{B-IW}(\alpha, \beta, a, b)$ & 1.6097 & 0.4046 & 22.0143 & 29.7617 \\
& $(2.498)$ & $(0.108)$ & $(21.432)$ & $(17.479)$ \\
$\operatorname{GE}-\mathrm{IW}(\alpha, \beta, a, b)$ & 1.3692 & 0.4776 & 27.6452 & 17.4581 \\
& $(2.017)$ & $(0.133)$ & $(14.136)$ & $(14.818)$ \\
$\operatorname{E}-\mathrm{IW}(\alpha, \beta, a)$ & 1.3692 & 0.4776 & 27.6452 & 17.4581 \\
& $(2.017)$ & $(0.133)$ & $(14.136)$ & $(14.818)$
\end{tabular}




\begin{tabular}{llll}
$\operatorname{T}-\operatorname{IW}(\alpha, \beta, a)$ & 1.9315 & 1.7435 & 0.0819 \\
& $(0.097)$ & $(0.076)$ & $(0.198)$ \\
$\operatorname{MO}-\operatorname{IW}(\alpha, \beta, a)$ & 2.3066 & 1.5796 & 0.5988 \\
& $(0.498)$ & $(0.16)$ & $(0.3091)$ \\
$\operatorname{IW}(\alpha, \beta)$ & 1.8705 & 1.7766 & \\
& $(0.112)$ & $(0.113)$ & \\
\hline
\end{tabular}

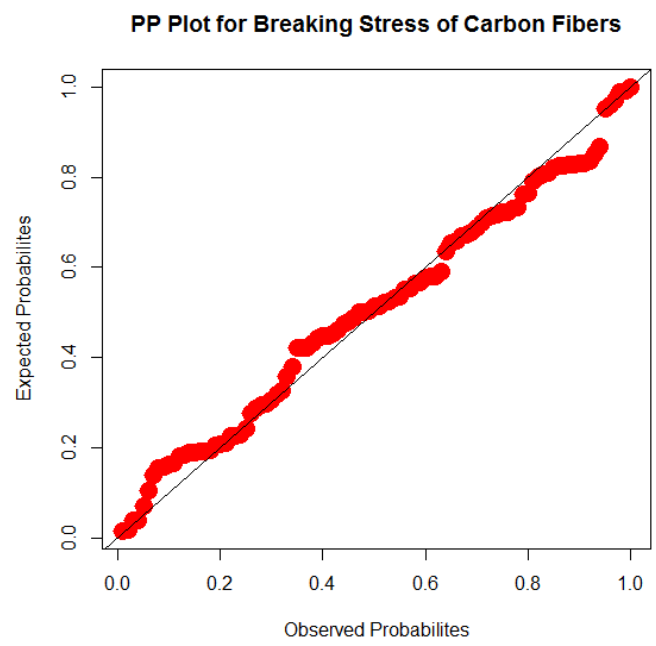

Histogram of Breaking Stress Data
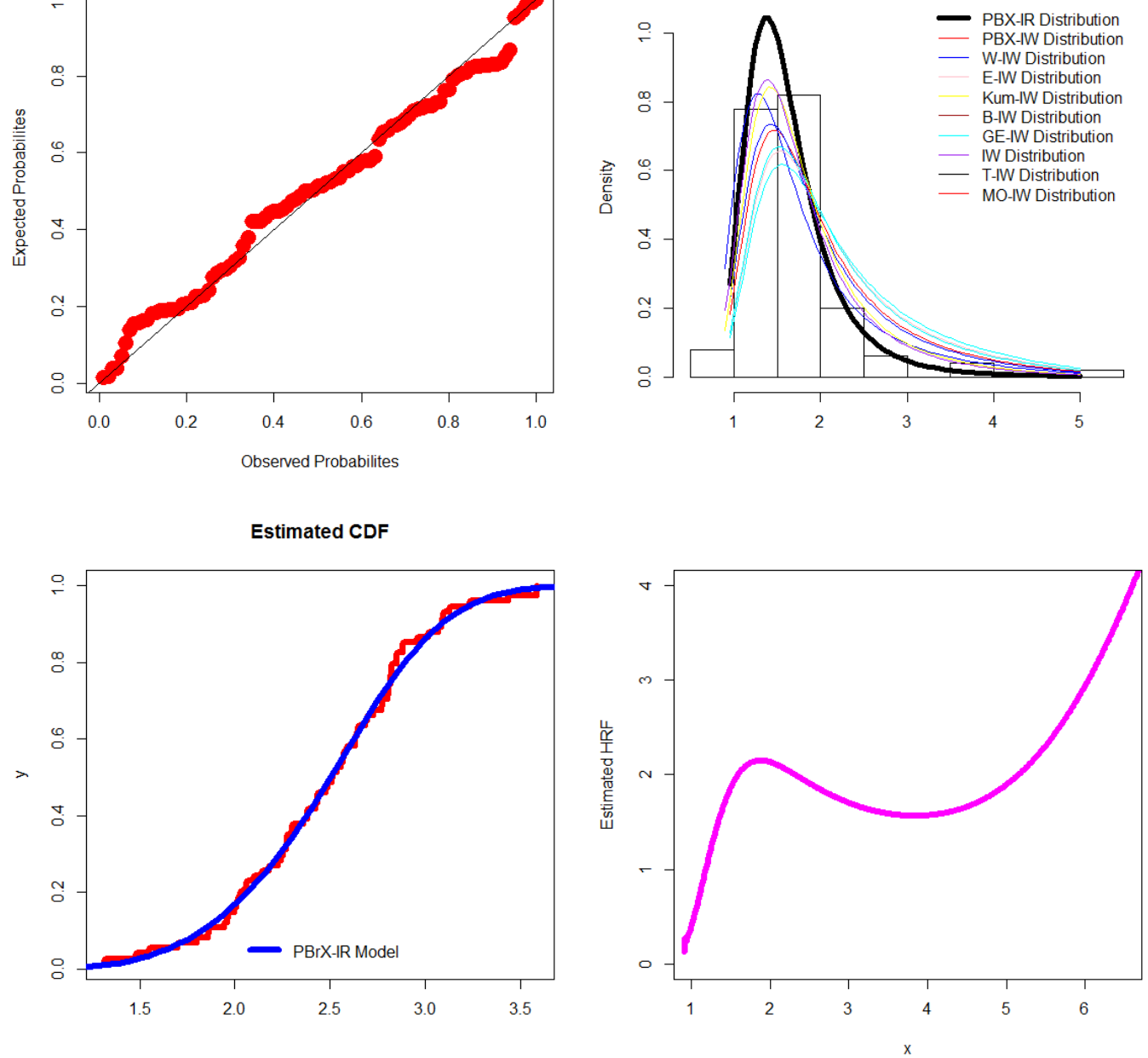


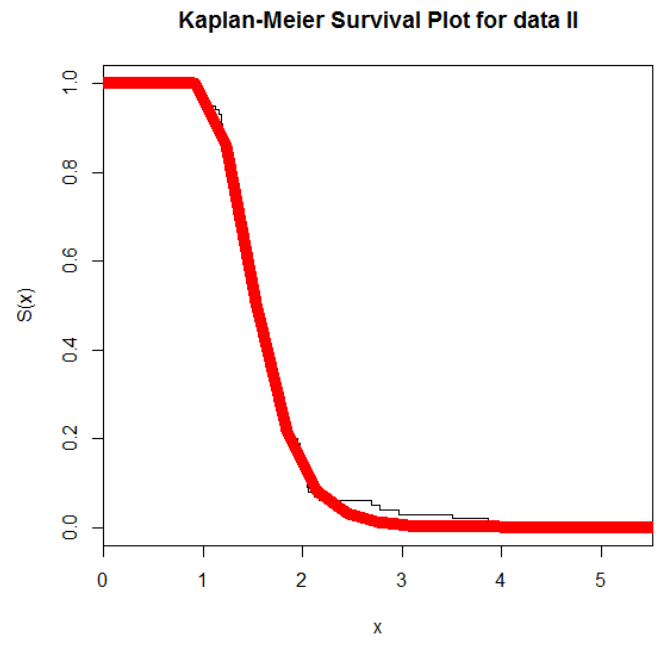

Figure 3: P-P plot, Estimated PDF, estimated CDF, estimated HRF and Kaplan-Meier Survival Plot S(x) for data set I.
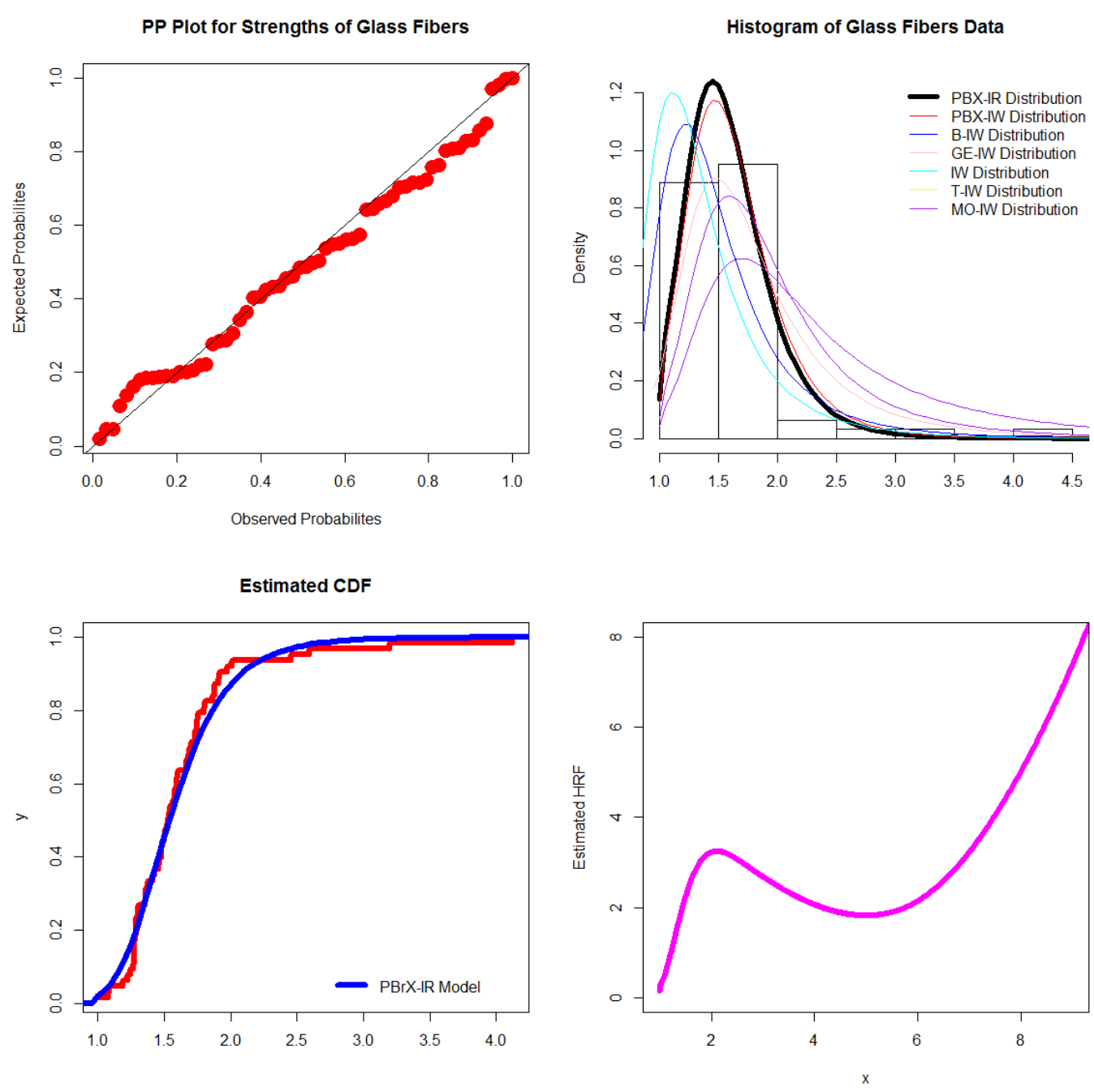


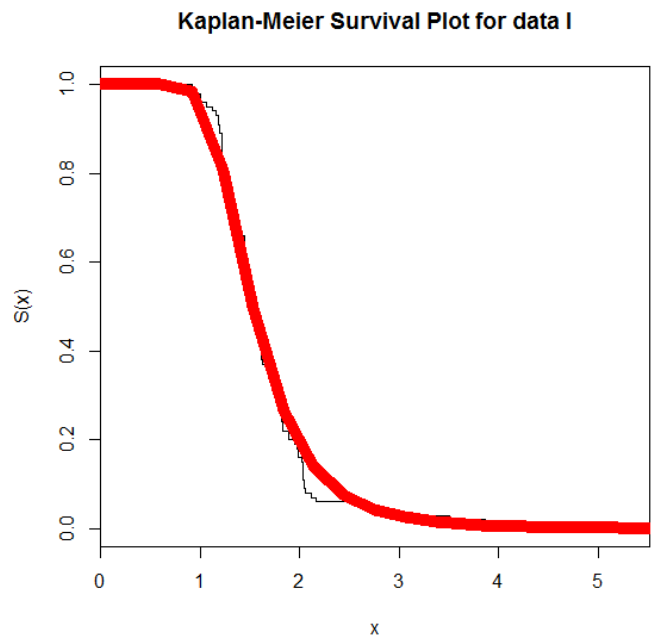

Figure 4: P-P plot, Estimated PDF, estimated CDF, estimated HRF and Kaplan-Meier Survival Plot S(x) for data set II.
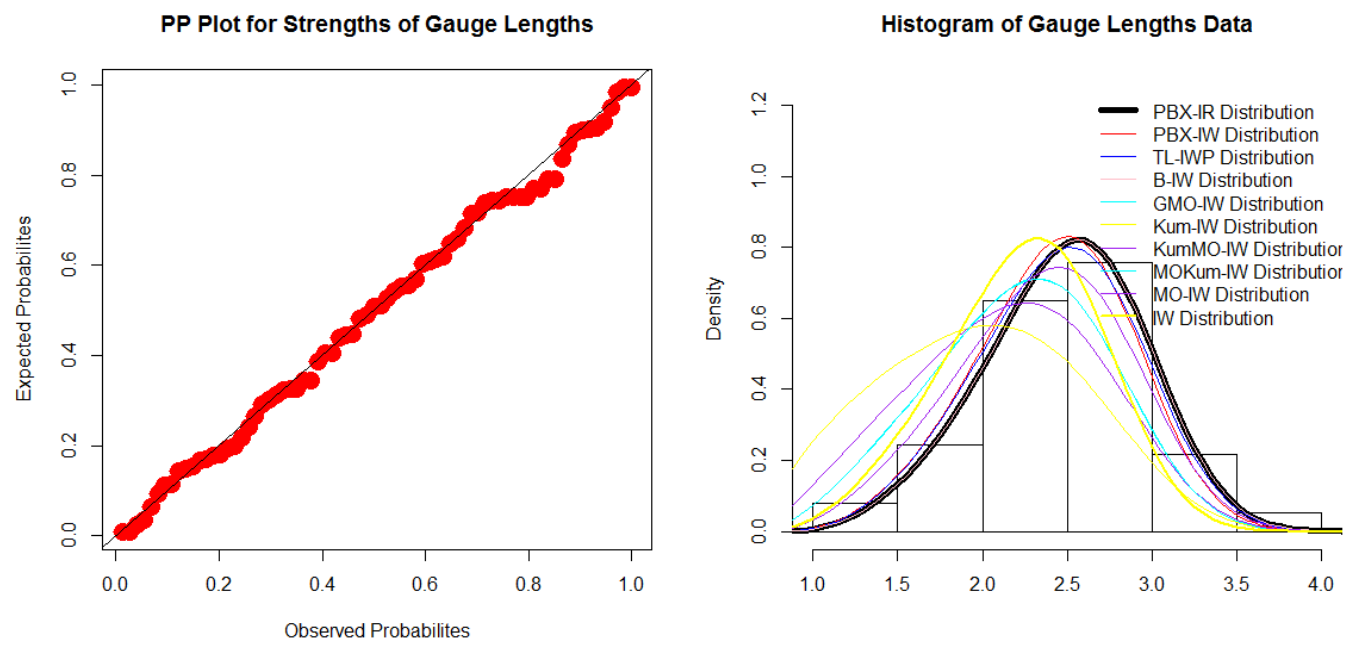

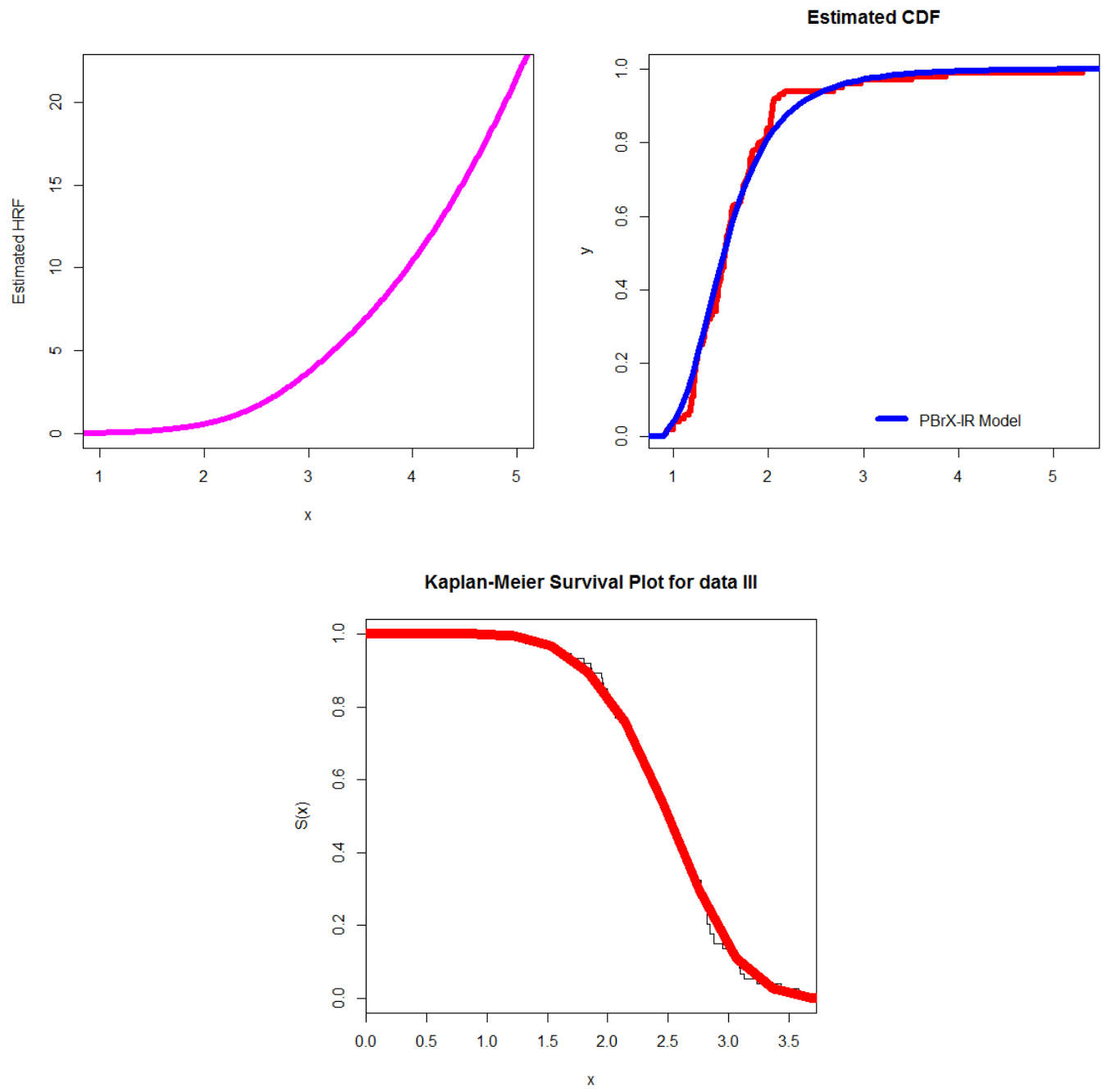

Figure 5: P-P plot, Estimated PDF, estimated CDF, estimated HRF and Kaplan-Meier Survival Plot S(x) for data set III.

Table 4: The statistics AIC;BIC;HQIC and CAIC values for glass fibre data. 


\begin{tabular}{ccccc}
\hline \hline Model & \multicolumn{4}{c}{ Measures } \\
\cline { 2 - 5 } & $A I C$ & $B I C$ & $H Q I C$ & $C A I C$ \\
\hline \hline PBX-IR & $\mathbf{4 9 . 1 3}$ & $\mathbf{5 5 . 6}$ & $\mathbf{5 1 . 7}$ & $\mathbf{4 9 . 5}$ \\
BrXI-IW & 49.58 & 58.15 & 52.9 & 50.3 \\
B-IW & 68.6 & 77.2 & 72.0 & 69.3 \\
GE-IW & 69.6 & 78.1 & 72.9 & 70.3 \\
IW & 97.7 & 102 & 99.4 & 97.9 \\
T-IW & 100.1 & 106.5 & 102.6 & 100.5 \\
MO-IW & 101.7 & 108.2 & 104.2 & 102.1 \\
\hline \hline
\end{tabular}

Table 5: MLEs and their standard errors for glass fibre data.

\begin{tabular}{ccccc}
\hline \hline Model & \multicolumn{4}{c}{ Estimates } \\
\hline \hline PBX-IR $(\lambda, \theta, \alpha)$ & 9.717 & 0.167 & 4.322 & \\
& 2.026 & 0.0154 & 0.0026 & \\
PBrX-IW $(\lambda, \theta, \alpha, \beta)$ & 4.492 & 19.9989 & 0.383 & 0.506 \\
& $(1.778)$ & $(9.241)$ & $(0.148)$ & $(0.109)$ \\
& & & & \\
B-IW $(\alpha, \beta, a, b)$ & 2.0518 & 0.6466 & 15.0756 & 36.9397 \\
& $(0.986)$ & $(0.163)$ & $(12.057)$ & $(22.649)$ \\
GE-IW $(\alpha, \beta, a, b)$ & 1.6625 & 0.7421 & 32.112 & 13.2688 \\
& $(0.952)$ & $(0.197)$ & $(17.397)$ & $(9.967)$ \\
T-IW $(\alpha, \beta, a)$ & 1.3068 & 2.7898 & 0.1298 & \\
& $(0.034)$ & $(0.165)$ & $(0.208)$ & \\
MO-IW $(\alpha, \beta, a)$ & 1.5441 & 2.3876 & 0.4816 & \\
& $(0.226)$ & $(0.253)$ & $(0.252)$ & \\
$\operatorname{IW}(\alpha, \beta)$ & 1.264 & 2.888 & & \\
& $(0.059)$ & $(0.234)$ & & \\
\hline \hline
\end{tabular}


Table 6: The statistics AIC; BIC; HQIC and CAIC values for gauge lengths data

\begin{tabular}{ccccc}
\hline \hline Model & \multicolumn{4}{c}{ Measures } \\
\cline { 2 - 5 } PBrX-IR & $\mathbf{1 0 7 . 9 9}$ & $\mathbf{1 1 4 . 9 1}$ & $\mathbf{1 1 0 . 7 5}$ & $\mathbf{1 0 8 . 3 4}$ \\
PBrX-IW & 109.91 & 119.13 & 113.59 & 110.49 \\
TL-IWP & 111.00 & 120.22 & 111.58 & 114.68 \\
B-IW & 112.63 & 121.84 & 113.21 & 116.30 \\
GMO-IW & 112.80 & 122.00 & 113.37 & 126.48 \\
Kw-IW & 113.68 & 122.82 & 114.25 & 117.36 \\
KumMO-IW & 113.30 & 124.82 & 114.18 & 117.90 \\
MOKum-IW & 113.19 & 124.68 & 114.07 & 117.78 \\
MO-IW & 115.06 & 121.96 & 115.40 & 117.81 \\
IW & 142.02 & 146.63 & 142.19 & 143.86 \\
\hline \hline
\end{tabular}

Table 7: MLEs and their standard errors (in parentheses) for gauge lengths data. 


\begin{tabular}{|c|c|c|c|c|c|}
\hline Model & \multicolumn{5}{|c|}{ Estimates } \\
\hline$\overline{\operatorname{PBX}-\operatorname{IR}(\lambda, \theta, \alpha)}$ & $\begin{array}{c}-1.376 \\
(1.655)\end{array}$ & $\begin{array}{c}0.748 \\
(0.322)\end{array}$ & $\begin{array}{c}2.143 \\
(0.084)\end{array}$ & & \\
\hline $\operatorname{PBrX}-\operatorname{IW}(\lambda, \theta, \alpha, \beta)$ & $\begin{array}{c}-1.433 \\
(1.945)\end{array}$ & $\begin{array}{c}0.976 \\
(0.968)\end{array}$ & $\begin{array}{c}1.999 \\
(0.639)\end{array}$ & $\begin{array}{c}1.774 \\
(0.878)\end{array}$ & \\
\hline $\mathrm{B}-\mathrm{IW}(\alpha, \beta, a, b)$ & $\begin{array}{c}37.179 \\
(33.510)\end{array}$ & $\begin{array}{c}0.242 \\
(0.377)\end{array}$ & $\begin{array}{c}2.039 \\
(1.015)\end{array}$ & $\begin{array}{c}5.857 \\
(1.813)\end{array}$ & \\
\hline $\operatorname{GMO}-\mathrm{IW}(\alpha, \beta, \lambda, \theta)$ & $\begin{array}{c}1.577 \\
(0.139)\end{array}$ & $\begin{array}{c}5.918 \\
(0.945)\end{array}$ & $\begin{array}{c}3.702 \\
(2.683)\end{array}$ & $\begin{array}{c}63.707 \\
(38.655)\end{array}$ & \\
\hline $\operatorname{Kum}-\mathrm{IW}(\alpha, \beta, a, b)$ & $\begin{array}{c}4.384 \\
(1.012)\end{array}$ & $\begin{array}{c}1.005 \\
(0.223)\end{array}$ & $\begin{array}{c}3.218 \\
(1.036)\end{array}$ & $\begin{array}{c}217.031 \\
(268.565)\end{array}$ & \\
\hline $\operatorname{KumMO-IW}(\alpha, \beta, \theta, a, b)$ & $\begin{array}{c}25.127 \\
(19.688)\end{array}$ & $\begin{array}{c}0.894 \\
(0.396)\end{array}$ & $\begin{array}{c}0.016 \\
(0.023)\end{array}$ & $\begin{array}{c}0.827 \\
(0.789)\end{array}$ & $\begin{array}{c}16.985 \\
(24.975)\end{array}$ \\
\hline MOKum-IW $(\alpha, \beta, \theta, a, b)$ & $\begin{array}{c}2.415 \\
(1.032)\end{array}$ & $\begin{array}{c}1.221 \\
(0.412)\end{array}$ & $\begin{array}{c}7.995 \\
(13.063)\end{array}$ & $\begin{array}{c}2.933 \\
(0.825)\end{array}$ & $\begin{array}{c}35.707 \\
(41.500)\end{array}$ \\
\hline $\operatorname{TL}-\operatorname{IWP}(\alpha, \beta, \lambda, \theta)$ & $\begin{array}{c}8.324 \\
(3.839)\end{array}$ & $\begin{array}{c}1.524 \\
(0.588)\end{array}$ & $\begin{array}{c}71.411 \\
(82.496)\end{array}$ & $\begin{array}{c}0.452 \\
(0.317)\end{array}$ & \\
\hline $\operatorname{MO}-\mathrm{IW}(\alpha, \beta, a)$ & $\begin{array}{c}1.419 \\
(0.109)\end{array}$ & $\begin{array}{c}8.031 \\
(0.764)\end{array}$ & $\begin{array}{c}80.338 \\
(62.007)\end{array}$ & & \\
\hline $\operatorname{IW}(\alpha, \beta)$ & $\begin{array}{c}4.110 \\
(0.323)\end{array}$ & $\begin{array}{c}2.169 \\
(0.065)\end{array}$ & & & \\
\hline
\end{tabular}

where $\hat{\ell}$ denotes the log-likelihood function evaluated at the MLEs, $\kappa$ is the number of model parameters and $n$ is the sample size. The model with minimum values for these statistics could be chosen as the best model to fit the data. All results are obtained using the R PROGRAM. Tables 2, 4 and 6 compare the PBX-IR model with other important competitive distributions. The PBX-IR model gives the lowest values for the AIC, BIC, HQIC and CAIC statistics (in bold values) among all fitted models to these data. So, it may be considered as the best model among them. Figure 3, 4 and 5, respectively, display the plots of estimated density for the proposed model and estimated CDF of the new model for the three data sets. These plots reveal that the proposed distribution yields a better fit than other nested and non-nested models for both data sets.

\section{Conclusions}

A new flexible extension of the IR model is proposed and studied. Some of its fundamental statistical properties are derived such as quantile, moments, incomplete moments and moment generating function. We assessed the performance of the maximum likelihood estimators via a simulation study. The importance of the new model is shown via two applications to real data sets. The new model is much better than other important competitive models (the Weibull Inverse Weibull, exponentiated Inverse Weibull, Kumaraswamy Inverse Weibull, beta Inverse Weibull, transmuted Inverse Weibull, gamma 
extended Inverse Weibull, Marshall-Olkin Inverse Weibull, Marshall-Olkin Kumaraswamy Inverse Weibull, generalized Marshall-Olkin Inverse Weibull, Kumaraswamy MarshallOlkin Inverse Weibull and Inverse Weibull distributions) based on three real data sets.

\section{References}

[1] Afify, A. Z., Yousof, H. M., Cordeiro, G. M., Ortega, E. M. M. and Nofal, Z. M. (2016). The Weibull Fréchet distribution and its applications. Journal of Applied Statistics, 43, 2608-2626.

[2] Ahmad, F., Ahmad, S.P. and Ahmed, A. (2014). Transmuted Inverse Rayleigh distribution: A generalization of the Inverse Rayleigh Distribution. Mathematical Theory and Modeling, 4, 1-9.

[3] Aryal, G. R. and Yousof, H. M. (2017). The exponentiated generalized-G Poisson family of distributions. Economic Quality Control, 32, 1-17.

[4] Aslam, M. and Jun, C. H. (2009). A group acceptance sampling plans for truncated life tests based on the inverse Rayleigh and log-logistic distributions, Pakistan Journal of Statistics, 25, 107-119.

[5] Barreto-Souza, W. M., Cordeiro, G. M. and Simas, A. B. (2011). Some results for beta Fréchet distribution. Communication in Statistics-Theory and Methods, 40, 798-811.

[6] Dey, S. (2012). Bayesian estimation of the parameter and reliability function of an Inverse Rayleigh distribution. Malaysian Journal of Mathematical Sciences 6, 113-124.

[7] Korkmaz, M. C. Yousof, H. M. and Ali, M. M. (2017). Some Theoretical and Computational Aspects of the Odd Lindley Fréchet Distribution, Journal of Statisticians: Statistics and Actuarial Sciences, 2, 129-140.

[8] Korkmaz, M. C., Yousof, H. M., Hamedani G. G. and Ali, M. M. (2018). The Marshall-Olkin generalized G Poisson family of distributions, Pakistan Journal of Statistics, 34, 251267.

[9] Kundu, D. and Raqab, M. Z. (2009). Estimation of $R=P(X<Y)$ for three-parameter Weibull distribution. Statistics and Probability Letters, 79, 1839-1846.

[10] Mahmoud, M. R. and Mandouh, R. M. (2013). On the transmuted Fréchet distribution. Journal of Applied Sciences Research, 9, 5553-5561.

[11] Mukerjee, S.P. and Saran, L.K. (1984). Bivariate inverse Rayleigh distributions in reliability studies. J. Ind. Statist. Assoc., 22, 23-31. 
[12] Nichols, M. D, Padgett, W. J. (2006). A Bootstrap control chart for Weibull percentiles. Quality and Reliability Engineering International, 22, 141-151.

[13] Ramos, M. W. A. Marinho, P. R. D. Cordeiro, G. M., Silva, R. V. da and Hamedani, G. G. (2015) The Kumaraswamy-G Poisson family of distributions, J. Stat. Theory Appl. 14, 222-239.

[14] Ristić, M. M. and Nadarajah, S. (2014). A new lifetime distribution, Journal of Statistical Computation and Simulation, 84, 135-150

[15] Smith, R. L. and Naylor, J. C. (1987). A comparison of maximum likelihood and Bayesian estimators for the three-parameter Weibull distribution. Applied Statistics, 36, 358-369.

[16] Soliman, A., Essam A. Amin and Alaa A. Abd-EI Aziz. (2010). Estimation and Prediction from Inverse Rayleigh Distribution Based on Lower Record Values. Applied Mathematical Sciences. 4, 3057-3066.

[17] Voda, V. Gh. (1972). On the Inverse Rayleigh Distributed Random Variable. Rep. Statis. App. Res. JUSE. 19, 13-21.

[18] Yousof, H. M., Afify, A. Z., Hamedani, G. G. and Aryal, G. (2017a). The Burr X generator of distributions for lifetime data. Journal of Statistical Theory and Applications, 16, 288-305.

[19] Yousof, H. M., Alizadeh, M., Jahanshahiand, S. M. A., Ramires, T. G., Ghosh, I. and Hamedani G. G. (2017b). The transmuted Topp-Leone G family of distributions: theory, characterizations and applications, Journal of Data Science. 15, 723-740.

[20] Yousof, H. M., Altun, E. and Hamedani, G. G. (2018a). A new extension of Frechet distribution with regression models, residual analysis and characterizations. Journal of Data Science, 16, 743-770.

[21] Yousof, H. M., Jahanshahi, S. M., Ramires, T. G Aryal, G. R. and Hamedani G. G. (2018b). A new distribution for extreme values: regression model, characterizations and applications. Journal of Data Science, 16, 677-706. 


\section{Appendix}

cdf_PBXIR - function(par,X)\{

lameda $=\operatorname{par}[1]$

theta $=\operatorname{par}[2]$

$\mathrm{a}=\operatorname{par}[3]$

$\mathrm{g}=\mathrm{b}^{*}(\mathrm{a} 2) * \mathrm{x}(-(2+1)) *(\exp (-((\mathrm{a} / \mathrm{x})(2))))$

$\mathrm{G}=\exp (-((\mathrm{a} / \mathrm{x})(2)))$

g.PBrX $=\left(\right.$ lameda* $\left(\left(2 *\right.\right.$ theta $\left.^{*} \mathrm{~g}^{*} \mathrm{G} /(1-\mathrm{G}) 3\right) * \exp (-(\mathrm{G} /(1-\mathrm{G})) 2) *(1-\exp (-(\mathrm{G} /(1-$

G))2))(theta-1) $) * \exp (-$ lameda*((1-exp(-(G/(1-G))2))theta) $)) /(1-\exp (-$ lameda $))$

G.PBrX $=(1-\exp (-$ lameda* $((1-\exp (-(G /(1-G)) 2))$ theta $))) /(1-\exp (-$ lameda $))$ return(G.PBX)

\}

pdf_PBXIR - function(par,x)\{

lameda $=\operatorname{par}[1]$

theta $=\operatorname{par}[2]$

$\mathrm{a}=\operatorname{par}[3]$

$\mathrm{g}=\mathrm{b}^{*}(\mathrm{a} 2) * \mathrm{x}(-(2+1)) *(\exp (-((\mathrm{a} / \mathrm{x})(2))))$

$\mathrm{G}=\exp (-((\mathrm{a} / \mathrm{x})(2)))$

$\mathrm{c}=\left(2 *\right.$ theta $\left.^{*} \mathrm{~g} * \mathrm{G} /(1-\mathrm{G}) 3\right) * \exp (-(\mathrm{G} /(1-\mathrm{G})) 2) *(1-\exp (-(\mathrm{G} /(1-\mathrm{G})) 2))($ theta- 1$)$

$\mathrm{C}=(1-\exp (-(\mathrm{G} /(1-\mathrm{G})) 2))$ theta

g.PBrX $=\left(\right.$ lameda* $\left(\left(2 *\right.\right.$ theta $\left.^{*} \mathrm{~g}^{*} \mathrm{G} /(1-\mathrm{G}) 3\right) * \exp (-(\mathrm{G} /(1-\mathrm{G})) 2) *(1-\exp (-(\mathrm{G} /(1-$

G))2))(theta-1))*exp(-lameda*((1-exp(-(G/(1-G))2))theta) $)) /(1-\exp (-$ lameda $))$

G.PBrX $=(1-\exp (-$ lameda* $((1-\exp (-(\mathrm{G} /(1-\mathrm{G})) 2))$ theta $))) /(1-\exp (-$ lameda $))$ return(g.PBX)

\}

$\mathrm{n}=20$;

\#lameda $=0.5 ;$ theta $=1.5 ; \mathrm{a}=2.5$

lameda $=1.5 ;$ theta $=0.5 ; \mathrm{a}=1.5$

$\mathrm{x}=\mathrm{Q}(\mathrm{u})$

fit $=$ goodness.fit $\left(p d f=\right.$ pdf_PBXIR, $c d f=c d f \_P B X I R$, starts $=c($ initial,initial,initial $)$, data $=x$, method $=$ "N", domain=c(0,Inf), mle=NULL)

fit\$mle

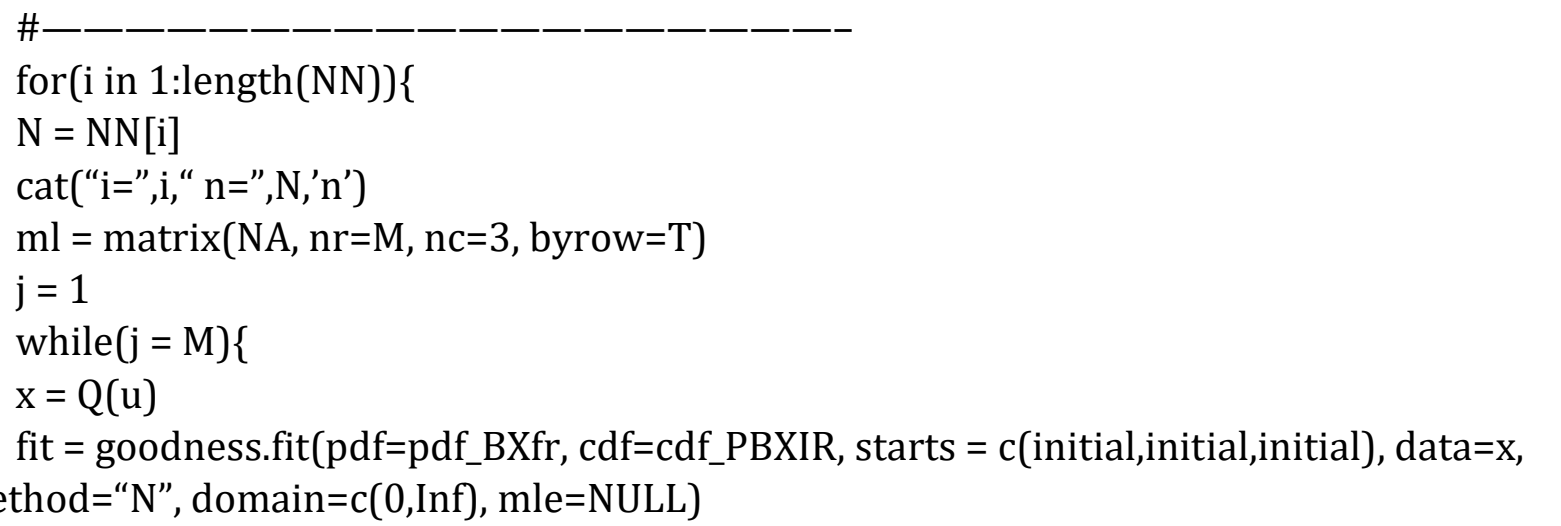


if(fit\$Convergence $==0$ ) \{

$\mathrm{ml}[\mathrm{j}]=$, fit $\$ \mathrm{mle}$

$\mathrm{j}=\mathrm{j}+1$

\}

\# for(k in 1:length $(\mathrm{ml}[, 1]))\{$

$\#$ if $\left(\mathrm{ml}[\mathrm{k}, 1] 1.5^{*}\right.$ lameda $) \mathrm{ml}[\mathrm{k}, 1]=\mathrm{NA}$

\# if(ml[k,2] 1.5*theta) $\mathrm{ml}[\mathrm{k}, 2]=\mathrm{NA}$

\# if(ml[k,3] 1.5*a) $\mathrm{ml}[\mathrm{k}, 3]=\mathrm{NA}$

\# \}

bias[i,]=apply((ml-pa), 2, FUN=mean, na.rm=TRUE)

$\operatorname{MSE}[\mathrm{i}]=,\operatorname{apply}((\mathrm{ml}-\mathrm{pa}) 2,2, \mathrm{FUN}=$ mean, na.rm=TRUE $)$

bias; MSE 